\title{
Anesthetic management for cesarean section in a patient receiving transplacental treatment of fetal tachyarrhythmia: a case report
}

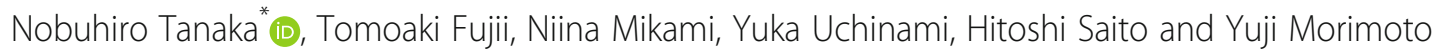

\begin{abstract}
Background: Although rare, long-lasting fetal tachyarrhythmia often leads to fetal heart failure and hydrops. Some mothers receive transplacental treatment of fetal tachyarrhythmia (TFT), which can potentially worsen maternal hypotension and bradycardia. Moreover, the use of rescue cardiovascular agents intraoperatively can worsen fetal tachycardia. However, reports of the anesthetic management of patients receiving TTFT are rare.

Case presentation: A 31-year-old woman who was receiving digoxin and sotalol for TTFT underwent planned elective cesarean section. The fetus had hypoplastic left heart syndrome, hydrops, and tachycardia. We used combined spinal-epidural anesthesia with a reduced dose of local anesthetic. We also employed a non-invasive continuous hemodynamic monitoring system. The mother's systolic blood pressure remained at $\geq 90 \%$ of the baseline value; intraoperative administration of rescue cardiovascular agents was not required.

Conclusions: We successfully anesthetized a woman for cesarean section, who was receiving TTFT for fetal tachyarrhythmia, using combined spinal-epidural anesthesia and non-invasive continuous hemodynamic monitoring.
\end{abstract}

Keywords: Cesarean section, ClearSight ${ }^{\mathrm{TM}}$ system, Fetal tachyarrhythmia, Non-invasive continuous hemodynamic monitoring, Transplacental anti-arrhythmic treatment

\section{Background}

Although fetal tachyarrhythmia is encountered in less than $0.1 \%$ of pregnancies [1], hydrops has been reported in up to $30-40 \%$ of fetuses with supraventricular tachycardia and in $7-43 \%$ of those with atrial flutter $[2,3]$. The perinatal mortality associated with fetal hydrops has been reported as $35 \%$ regardless of treatment [4]. Several studies have described the efficacy of digoxin, sotalol, flecainide, and amiodarone for transplacental treatment of fetal tachyarrhythmia (TTFT) $[5,6]$; however, a standard protocol has yet to be established. A prospective multicenter trial to establish a standardized protocol for TTFT is currently underway in Japan in which mothers

\footnotetext{
* Correspondence: dorami1105@hotmail.com

Department of Anesthesiology and Critical Care Medicine, Hokkaido University Graduate School of Medicine, Kita-15, Nishi-7, Kita-ku, Sapporo 060-8638, Japan
}

are administered digoxin monotherapy, digoxin with sotalol, or digoxin with flecainide [4]. Hence, we believe that the combination of digoxin and sotalol used in this case will become mainstream for TTFT in the future.

TTFT does not limit the mode of delivery to vaginal delivery or cesarean section, but reports describing the anesthetic management of patients receiving TTFT are rare. A single report describes the anesthetic management of a woman undergoing a cesarean section while receiving TTFT, but high-dose amiodarone was used in that case [7]. To the best of our knowledge, the present report is the first to describe the anesthetic management of a parturient receiving digoxin and sotalol for TTFT.

In planning the anesthetic management, we were concerned that spinal anesthesia may cause maternal hypotension and bradycardia, both of which may be exacerbated by the anti-arrhythmic drugs. Additionally, the 
intraoperative use of rescue cardiovascular agents, such as ephedrine and atropine, could worsen fetal tachycardia. Thus, continuous hemodynamic monitoring seemed warranted in this case. Here, we describe the successful anesthetic management of cesarean section in a mother who was taking digoxin and sotalol for TTFT.

\section{Case presentation}

A 31 -year-old woman $(161 \mathrm{~cm}, 59.2 \mathrm{~kg}, 48 \mathrm{~kg}$ before pregnancy, gravida 1 , para 0 , abortus 0 ) who was receiving digoxin and sotalol for TTFT was scheduled to undergo an elective cesarean section.

Fetal tachyarrhythmia was first identified at 26 weeks and 0 days of gestation, and the fetus was found to have hypoplastic left heart syndrome (HLHS), hydrops, and tachycardia with a heart rate (HR) of around 250 beats per minute (bpm). The mother received digoxin $(0.75$ $\mathrm{mg} /$ day) and sotalol $(320 \mathrm{mg} /$ day $)$ for TTFT from 26 weeks and 1 day of gestation to the day of surgery. The fetal tachycardia and hydrops immediately improved, and the fetal HR stabilized at $140-150 \mathrm{bpm}$. The maternal HR was in the $60 \mathrm{~s}$ before starting TTFT and in the $50 \mathrm{~s}$ after starting TTFT. The maternal blood concentration of digoxin remained at $1.3-1.5 \mathrm{ng} / \mathrm{mL}$, and she experienced no adverse events. The cesarean section was scheduled at 37 weeks and 5 days of gestation to facilitate the treatment schedule of the infant's HLHS.

Combined spinal-epidural anesthesia (CSEA) was chosen for the cesarean section. The ClearSight ${ }^{\mathrm{mit}}$ system (Edwards Lifesciences Corp., Irvine, CA, USA) was used in addition to the standard hemodynamic monitoring. The ClearSight ${ }^{\mathrm{mix}}$ finger cuff was placed on the mother's left middle finger, and a non-invasive blood pressure (NIBP) monitor (IntelliVue MP70; Philips Electronics Japan Corp., Tokyo, Japan) was placed on her right upper arm. Intravenous access was established using a $20 \mathrm{G}$ cannula. The patient was given $400 \mathrm{~mL}$ of $6 \%$ hydroxyethyl starch 130/0.4/9 (Voluven ${ }^{\circ}$, Fresenius Kabi, Bad Hamburg, Germany) from when she arrived in the operating theater until the start of the operation. CSEA was performed with the patient in the left lateral decubitus position. An epidural catheter was inserted at the T12-L1 level using an 18G Tuohy needle. Spinal anesthesia was performed at the L3-4 levels using a 27 G Quincke needle, and 0.5\% hyperbaric bupivacaine $9 \mathrm{mg}$ and fentanyl $15 \mathrm{mcg}$ were administered intrathecally. The surgery began after confirming the loss of cold sensation up to the T8 dermatome.

We sought to maintain the mother's systolic blood pressure (SBP) at $\geq 90 \%$ of the baseline value. If her SBP fell below $90 \%$ of the baseline, we planned to administer phenylephrine if her HR was $>60 \mathrm{bpm}$ or ephedrine 4$8 \mathrm{mg}$ if her HR was $<60 \mathrm{bpm}$. Her baseline blood pressure was $116 / 71 \mathrm{mmHg}$ by the ClearSight ${ }^{\mathrm{Tm}}$ system, and her SBP as measured by the ClearSight ${ }^{\mathrm{Tm}}$ system stayed over $115 \mathrm{mmHg}$ until the infant's birth. The SBP value measured by the NIBP cuff was somewhat lower than that measured by the ClearSight ${ }^{\text {tix }}$ system, but the difference was within $10 \%$.

The duration of anesthesia was $94 \mathrm{~min}$, and the duration of surgery was $52 \mathrm{~min}$ (Fig. 1). The total amount of fluid administered was $1055 \mathrm{~mL}$, which comprised crystalloid $655 \mathrm{~mL}$ and colloid $400 \mathrm{~mL}$. The total blood loss, including amniotic fluid, was $745 \mathrm{~mL}$ and the mother produced $200 \mathrm{~mL}$ of urine. The infant's birth weight was $2618 \mathrm{~g}$, and the Apgar scores were 8, 8, and 8 at 1, 3, and $5 \mathrm{~min}$, respectively. The infant's initial umbilical artery $\mathrm{pH}$ was 7.252. The fetal HR was in the $140 \mathrm{~s}$ before entering the operating theater, and the infant's HR was in the $130 \mathrm{~s}$ upon admission to the neonatal intensive care unit. Sotalol was administrated to the infant from the first day of life to prevent tachyarrhythmia and the infant's HR remained stable at $120-150 \mathrm{bpm}$.

The infant underwent a pulmonary artery banding operation at 4 days of age, the Norwood operation at 20 days of age, and the bidirectional Glenn operation at 3 months of age. The mother was found to have an elevated $\mathrm{D}$-dimer level $(21.66 \mu \mathrm{g} / \mathrm{mL})$ on postoperative day (POD) 7. Lower extremity venous ultrasonography and contrast-enhanced computed tomography were performed to rule out deep vein thrombosis; however, there were no abnormal findings and she was discharged home on POD 10.

\section{Discussion}

To the best of our knowledge, this is the first case report describing the anesthetic management for cesarean section in a parturient receiving digoxin and sotalol for TTFT. Amiodarone is the least preferred choice for TTFT due to its side effects such as neonatal hypothyroidism [8]. The first-line drug of choice for treating fetal tachycardia remains controversial, but digoxin is usually used, followed by sotalol or flecainide either alone or in combination with digoxin. In cases with fetal hydrops, the combination of digoxin and sotalol is accepted as first-line therapy [4]. Sotalol is a beta-adrenoceptor blocker that also has class III anti-arrhythmic properties. Although sotalol is less well reported in the literature, its use is increasing $[5,6]$.

We carefully considered the potential anesthetic risks in this case. Firstly, the bradycardia that may occur following spinal anesthesia could have been exacerbated by the anti-arrhythmic drugs the mother was taking. Although digoxin was not found to increase the risk of bradycardia after neuraxial anesthesia in a previous study, beta-adrenoceptor blockers are suggested as a risk factor of moderate bradycardia (HR of $40-50 \mathrm{bpm}$ ) [9]. 


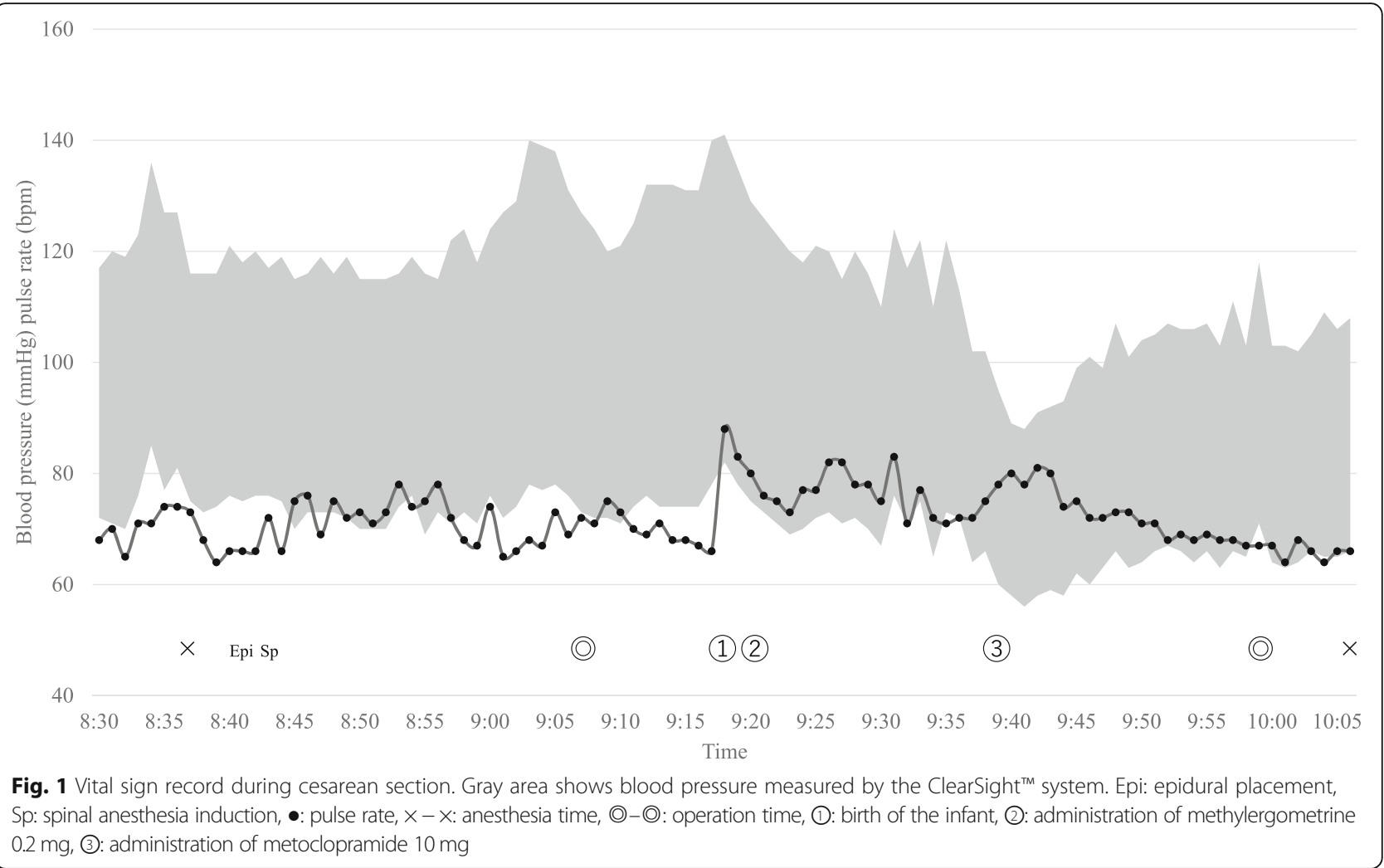

Another study found a higher incidence of bradycardia after a neuraxial block when the analgesic level was at or above the T4 dermatome [10].

Secondly, we sought to minimize the use of atropine and ephedrine intraoperatively since these drugs may worsen fetal tachycardia. We also considered the management of maternal hypotension because phenylephrine could have caused maternal bradycardia requiring atropine treatment [11]. Moreover, ephedrine's beta-adrenergic effect may be attenuated by the sotalol. In order to minimize maternal hypotension, bradycardia, and the need for rescue cardiovascular agents, we decided to employ a sensitive, continuous hemodynamic monitoring device in addition to standard monitoring.

The most common definitions of hypotension during cesarean section are either SBP $<80 \%$ of the baseline value or $<100 \mathrm{mmHg}$ [12]; however, current guidelines recommend that SBP should be maintained at $\geq 90 \%$ of the baseline after induction of spinal anesthesia [12]. To avoid maternal and fetal adverse effects, we elected to maintain the mother's SBP at $\geq 90 \%$ of the baseline. Similarly, we decided to use an HR cutoff of $60 \mathrm{bpm}$ to decide whether to treat intraoperative hypotension with either phenylephrine or ephedrine according to a previous study [13]. Fortunately, the mother's SBP remained above $115 \mathrm{mmHg}$ and $\geq 90 \%$ of the baseline, and no rescue cardiovascular agents were required before the infant's birth.
The relatively low analgesic level (T8) likely accounted for the mother's stable hemodynamics. We used hyperbaric bupivacaine $9 \mathrm{mg}$, which is slightly lower than the recommended dose of $10 \mathrm{mg}$ according to Harten's dose chart $[14,15]$. We were also concerned about the potential maternal bradycardia associated with the use of intrathecal opioids, such as morphine [16]. We elected to use fentanyl, which was not associated with maternal bradycardia in a previous report [17]. The mother did not complain of any pain during the surgery.

In recent years, studies have reported the use of the ClearSight $^{\mathrm{Tx}}$ (previously called Nexfin ${ }^{\mathrm{Tn}}$ ) continuous non-invasive hemodynamic monitoring system during cesarean section $[13,18]$. These reports demonstrated that use of the ClearSight ${ }^{\mathrm{Tw}}$ system helped minimize the incidence of intraoperative maternal hypotension. In the present case, there were small differences between the blood pressure values obtained by the NIBP cuff and the ClearSight $^{\text {tm }}$ system. Indeed, another study found large limits of agreement between direct radial arterial pressure and the ClearSight ${ }^{\mathrm{tm}}$ system [19]. Accordingly, invasive continuous blood pressure monitoring may still be superior if either the mother or fetus is hemodynamically unstable.

\section{Conclusions}

As care improves for infants born with congenital cardiac abnormalities, the use of TTFT will likely increase. 
There are several important anesthetic considerations in patients who are receiving this type of treatment, including the risk of maternal bradycardia and hypotension, as well as the potential risks of using rescue cardiovascular agents. In this case, we successfully provided a safe and effective anesthetic using CSEA and non-invasive continuous hemodynamic monitoring in a patient undergoing cesarean section who was receiving a combination of digoxin and sotalol for TTFT.

\section{Abbreviations \\ bpm: Beats per minute; CSEA: Combined spinal-epidural anesthesia; HLHS: Hypoplastic left heart syndrome; HR: Heart rate; NIBP: Non-invasive blood cuff pressure; SBP: Systolic blood pressure; TTFT: Transplacental treatment of fetal tachyarrhythmia}

\section{Acknowledgements}

We would like to thank Editage (http://www.editage.jp) for English language editing.

\section{Funding}

None.

\section{Availability of data and materials}

Not applicable.

\section{Authors' contributions}

NT drafted the manuscript and managed the patient's anesthesia. TF also managed the patient's anesthesia. YM provided substantial advice for the preparation and revision of the manuscript. All authors have reviewed and approved the final manuscript.

\section{Ethics approval and consent to participate} Not applicable.

\section{Consent for publication}

Written informed consent was obtained from the patient for the publication of this case report.

\section{Competing interests}

The authors declare that they have no competing interests.

\section{Publisher's Note}

Springer Nature remains neutral with regard to jurisdictional claims in published maps and institutional affiliations.

Received: 11 February 2019 Accepted: 24 April 2019

Published online: 07 May 2019

\section{References}

1. Sridharan S, Sullivan I, Tomek V, Wolfenden J, Skovranek J, Yates R, et al. Flecainide versus digoxin for fetal supraventricular tachycardia: comparison of two drug treatment protocols. Heart Rhythm. 2016;13:1913-9.

2. Krapp M, Kohi T, Simpson JM, Sharland GK, Katalinic GU. Review of diagnosis, treatment, and outcome of fetal atrial flutter compared with supraventricular tachycardia. Heart. 2003;89:913-7.

3. Cuneo BF, Strasburger JF. Management strategy for fetal tachycardia. Obset Gynecol. 2000;96:575-81.

4. Miyoshi T, Maeno Y, Sago H, Inamura N, Yasukochi S, Kawataki M, et al. Antenatal antiarrhythmic treatment for fetal tachyarrhythmias: a study protocol for a prospective multicentre trial. BMJ Open. 2017;7:e016597.

5. Hill GD, Kovach JR, Saudek DE, Singh AK, Wehrheim K, Frommelt MA. Transplacental treatment of fetal tachycardia: a systematic review and metaanalysis. Prenat Diagn. 2017;37:1076-83.

6. Alsaied T, Baskar S, Fares M, Alahdab F, Czosek RJ, Murad MH, et al. First-line antiarrhythmic transplacental treatment for fetal tachyarrhythmia: a systematic review and meta-analysis. J Am Heart Assoc. 2017;6:e007164.
7. Fulgencio JP, Hamza J. Anaesthesia for caesarean section in a patient receiving high dose amiodarone for fetal supraventricular tachycardia. Anaesthesia. 1994;49:406-8

8. Bartalena L, Bogazzi F, Braverman LE, Martino E. Effects of amiodarone administration during pregnancy on neonatal thyroid function and subsequent neurodevelopment. J Endocrinol Investig. 2001;24:116-30.

9. Lesser JB, Sanborn KV, Valskys R, Kuroda M. Severe bradycardia during spinal and epidural anesthesia recorded by an anesthesia information management system. Anesthesiology. 2003;99:859-66.

10. Kyokong O, Charuluxananan S, Sriprajittichai P, Poomseetong T, Naksin P. The incidence and risk factors of hypotension and bradycardia associated with spinal anesthesia. J Med Assoc Thail. 2006;89:58-64

11. Lee A, Ngan K, Warwick D, Gin T. A quantitative, systematic review of randomized controlled trials of ephedrine versus phenylephrine for the management of hypotension during spinal anesthesia for cesarean delivery. Anesth Analg. 2002;94:920-6.

12. Kinsella SM, Carvalho B, Dyer RA, Fernando R, McDonnell N, Mercier FJ, et al. International consensus statement on the management of hypotension with vasopressors during caesarean section under spinal anaesthesia. Anaesthesia. 2018:73:71-92.

13. Sng BL, Wang H, Assam PN, Sia AT. Assessment of an updated doublevasopressor automated system using Nexfin ${ }^{\mathrm{TM}}$ for the maintenance of haemodynamic stability to improve peri-operative outcome during spinal anaesthesia for cesarean section. Anaesthesia. 2015;70:691-8.

14. Harten JM, Boyne I, Hannah P, Varveris D, Brown A. Effets of a height and weight adjusted dose of local anaesthetic for spinal anaesthesia for elective caesarean section. Anaesthesia. 2005;60:348-53.

15. Subedi A, Tripathi M, Bhattarai BK, Gupta PK, Pokharel K, Regmi MC. The effect of height and weight adjusted dose of intrathecal hyperbaric bupivacaine for elective caesarean section. J Nepal Med Assoc. 2011;51:1-6.

16. Somboonviboon W, Kyokong O, Charuluxananan S, Narasethakamol A. Incidence and risk factors of hypotension and bradycardia after spinal anesthesia for cesarean section. J Med Assoc Thail. 2008:91:181-7.

17. Khezri MB, Rezaei M, Reihany MD, Javadi EHS. Comparison of postoperative analgesic effect of intrathecal clonidine and fentanyl added to bupivacaine in patients undergoing cesarean section: a prospective randomized doubleblind study. Pain Res Treat. 2014:2014:513628.

18. Juri T, Suehiro K, Kimura A, Tanaka K, Yamada T, Mori T, et al. Impact of noninvasive continuous blood pressure monitoring on maternal hypotension during cesarean delivery: a randomized controlled study. J Anesth. 2018;32: 822-30

19. Weiss E, Gayat E, Dumans-Nizard V, Le Guen M, Fischler M. Use of the Nexfin $^{\text {TM }}$ device to detect acute arterial pressure variations during anaesthesia induction. BJA. 2014:113:52-60.

\section{Submit your manuscript to a SpringerOpen ${ }^{\circ}$ journal and benefit from:}

- Convenient online submission

- Rigorous peer review

- Open access: articles freely available online

High visibility within the field

- Retaining the copyright to your article

Submit your next manuscript at $>$ springeropen.com 